\title{
Os Arquivos Brasileiros de Nutrição: uma revisão sobre produção científica em nutrição no Brasil (1944 a 1968)
}

\author{
Arquivos Brasileiros de Nutrição: \\ a review of scientific research on nutrition \\ in Brazil from 1944 to 1968
}

Francisco de Assis Guedes de Vasconcel os 1

\footnotetext{
1 Departamento de Nutrição, Centro de Ciências da Saúde, Universi dade Federal de Santa Catarina. Campus Universitário Trindade, Florianópolis,SC 88040-900, Brasil.
}

Abstract This study reviews 209 original articles published in the journal Arquivos Brasilei ros de Nutrição (1944/1968), a periodical edited by Josué de Castro, physician, specialist in nutrition, and founder-director of the Institute of Nutrition at the University of Brazil (now the Federal University of Rio de Janeiro). Our methodology was based on quantitative and qualitative analyses, aimed at summarizing both the topic itself and the authors' backgrounds. Results showed that 134 of the articles (64\%) adopted a biological perspective to nutrition, mostly focusing on laboratory research concerning the chemical composition and nutritional value of Brazilian foodstuffs. On the other hand, 75 articles (36\%) took a social perspective, testifying to the first efforts by Brazilian nutritional experts to create and improve specific methodological tools for investigating our population's nutritional conditions, thereby hel ping to consolidate the field of nutrition in the country.

Key words Nutrition; History of Nutrition; Serial Publications; Periodicals

Resumo Este trabalho consiste em uma revi são de 209 arti gos origi nais publicados pel os Arquivos Brasileiros de Nutrição (1944/1968), um periódico editado pelo médi co-nutról ogo Josué de Castro, diretor-fundador do Instituto de Nutrição da Universi dade do Brasil (atual Universi dade Federal do Rio de Janei ro). A metodologia utilizada baseou-se na realização de uma análise quanti-qualitativa, buscando sumariar tanto os conteúdos temáti cos abordados, como o perfil dos autores dos artigos. Os resultados deste estudo revelam, por um lado, que 134 destes artigos (64\%) foram inseridos dentro da perspectiva bi ológica da nutrição e correspondem, na maioria, a pesquisas laboratoriais sobre a composi ção química e o valor nutricional de alimentos naci onais. Por outro lado, a anál ise dos 75 arti gos (36\%) incluídos na perspectiva social atesta o esforço dos primeiros nutról ogos brasil eiros em construir eaperfei çoar os instrumentos metodol ógicos específicos para investigar as condições nutricionais de nossa população, bem como em contribuir para a consolidação do campo da nutrição no Brasil.

Palavras-chave Nutrição; História da Nutrição; Publicações Seriadas; Periódicos 
Introdução

Nos últimos vinte anos, embora muito limitadas, foram realizadas importantes investigações abordando diferentes aspectos acerca do processo de constituição do campo da nutrição no Brasil, considerada como disci plina, política social e/ ou profissão (Castro, 1977; Coimbra et al., 1982; L'Abbate, 1982, 1988; Natal, 1982; Bosi, 1988, 1995; Santos, 1988; Vasconcelos, 1988; Prado, 1993; Lima, 1997; Magalhães, 1997).

Nessas distintas investigações, são sempre observadas referências ao periódico Arquivos Brasi lei ros de Nutri ção, considerado uma das principais fontes documentais da história desse campo específico do saber no Brasil. A publicação dessa revista científica, que teve início em maio de 1944, ainda no transcurso do chamado Estado Novo e da Segunda Guerra Mundial, constitui uma das inúmeras idealizações da trajetória político-intelectual do eminente médico-nutrólogo pernambucano Josué de Castro (1908-1973) (Coimbra et al., 1982; L'Abbate, 1988). Por meio de um convênio estabelecido entre o Serviço Técnico da Alimentação Nacional (STAN) - órgão da Coordenação da Mobilização Econômica, instituída pelo Presidente Getúlio Vargas, em setembro de 1942, em decorrência da Segunda Guerra Mundial - ea Nutrition Foundation, de Nova Iorque - órgão norte-americano responsável pela publicação da concei tuada Nutrition Reviews - - J osué de Castro criou os Arquivos Brasileiros de Nutrição com os seguintes objetivos: a) "contribuir com a difusão de conheci mentos seguros da Ciência da Nutrição para a formação deste corpo denutricionistas, especializados em quai squer dos diferentes setores da matéria" e b) "dar um conhecimento mais efetivo e mais amplo dos resul tados dos trabalhos edos estudos realizados no Brasil no campo da Nutri ção" (Castro, 1944: 5-6). Ainda em agosto de 1944, a publicação dos Arquivos, cuja periodicidade deveria ser mensal, foi interrompida após a edição do quarto número do seu primeiro volume, em razão da extinção do STAN e da Coordenação de Mobilização Econômica. Em novembro de 1946, os Arqui vos voltaram a ser editados sob a responsabilidade do então Instituto de Nutrição da Universidade do Brasil (Inub), atual Instituto de Nutrição Josué de Castro da Universidade Federal do Rio de Janeiro (Arquivos Brasileiros de Nutrição, 1946; Castro, 1946). Segundo algumas análises, até dezembro de 1968, mês de circulação do último volume do periódico, dentro e fora deste espaço acadêmico-institucional, os Arquivos constituíram-se no mais importante veículo de difusão do saber científico em alimentação produzido no País (Castro, 1946; Coimbra et al., 1982; L'Abbate, 1988).

Entre as investigações citadas acima, consideramos que apenas duas, apesar de suas limitações, realizaram revisões críticas específicas sobre os Arqui vos Brasileiros de Nutrição. O estudo de Coimbra et al. (1982) sobre a História da Alimentação Escolar no Brasil, uma das primeiras e mais completas das sistematizações sobre este tema específico, apresenta importantes informações e comentários a respeito daquele periódico. L'Abbate (1982, 1988), por sua vez, ao estudar as Políticas deAlimentação eNutrição no Brasil, também dedica uma parte de sua atenção à revisão bibliográfica narrativa do saber científico que foi produzido pelos intelectuais do campo da nutrição e divulgado através dos Arqui vos.

Assim sendo, o presente estudo tem como objetivo geral realizar uma reflexão acerca da produção científica no campo da nutrição no Brasil, enfocando os artigos originais publicados nos Arqui vos Brasileiros de Nutrição, no período de 1944 a 1968. Quanto aos objetivos específicos, a proposta desse trabal ho é: a) realizar uma análise quantitativa dos artigos publicados; b) traçar um perfil dos autores dos artigos no que se refere a suas identidades profissionais, seus vínculos institucionais e seus campos específicos de conhecimento; c) identificar os conteúdos temáticos abordados pelos artigos e d) construir subsídios para uma reflexão sobre a trajetória do campo da nutrição em saúde pública no Brasil.

\section{Metodologia}

O universo do nosso estudo foi constituído por um total de 209 trabalhos publicados na seção de artigos originais dos 24 volumes ( 76 fascículos) dos Arquivos Brasilei ros de Nutrição, editados no período de maio de 1944 a dezembro de 1968. Cabe ressaltar que, de acordo com as principais condições de publicação dos Arquivos (instruções que não se alteraram ao longo da trajetória do periódico), naquela seção seriam aceitos para publicação trabal hos inéditos de autores nacionais sobre nutrologia, que deveriam versar, de modo original, sobre os temas a que se propuseram; deveriam, ainda, apresentar resumo em portuguêse, se possível, em inglês e espanhol, seguindo as normas bibliográficas do Index Medicus. Destacamos, também, que os Arquivos se subdividiam nas seguintes seções seqüenciais: 1a) Grandes Vultos da Nutrologia, na qual, a cada número, por meio de fotografia e de uma síntese biográfica, 
a revista prestava homenagem a uma pessoa notável dentro do cenário universal ou nacional da nutrição; 2â) Editorial, onde os dirigentes da revista apresentavam abordagens conjunturai s sobre os mais di versifi cados temas; 3a) Artigos Originais, que foram objetos de nossa reflexão; 4ạ) Recentes Aquisições da Nutrologia, composta por resenhas dos artigos publicados na Nutrition Reviews, quase sempre a seção mais extensa do periódico; 5ạ) Resenha Nacional de Nutrição, composta por resenhas de artigos e de outras modalidades de publicações editadas no Brasil; 6ạ) Transcrições, composta por artigos publicados anteriormente em outros periódicos; 7ạ) Cursos e Conferências, onde são apresentadas breves informações, resumos, programas, agendas e/ ou currículos de eventos científicos, e 8a) Notas e Informações, apresentação de rel eases sobre uma miscelânea de temas relacionados ao campo da nutrição. Tais seções, com exceção das três últimas, mantiveram-se sem alteração do primeiro ao último volume do periódico.

A opção por investigar apenas a seção Artigos Originais dos Arquivos representa, antes de tudo, um recorte metodológico, uma via de aproximação para melhores aprofundamento e compreensão sobre a produção científica em nutrição, ao longo do extenso período analisado.

Cada artigo original (nossa unidade de análise) foi examinado conforme um roteiro de investigação construído para possibilitar tanto a anál ise quantitativa e de categorização dos artigos por volume editado, como a análise das características dos autores envolvidos. Dessa forma, além da elaboração de listagem dos 209 artigos, conforme normas bibliográficas (nome de autores, ano, título, nome do periódico, volume, número, páginas inicial e final e mês), cada artigo foi categorizado de acordo com os seguintes atributos: número de autores; número de páginas; apresentação de referências bibliográficas e outras condições de publicação definidas pelo periódico; vínculo institucional e identidade profissional do(s) autor(es); natureza da pesquisa que serviu de base para o artigo e conteúdo temático abordado.

Na seqüência dos procedimentos metodológicos adotados, os artigos foram categorizados dentro de duas modalidades de estudos: investigações de natureza biológica (que chamaremos de perspectiva biológica) e investigações de natureza social (que chamaremos de perspectiva social). A rigor, tal procedimento foi adotado procurando-se seguir tanto as finalidades, como a estrutura de organização do Inub, idealizadas por Josué de Castro. Desse modo, os artigos categorizados dentro da perspectiva biológica originam-se e/ ou atendem às finalidades de três das quatro seções organizativas do então Inub: a Seção de Pesquisas Biológicas, a Seção de Patologia da Nutrição e, de certa forma, a Seção de Educação Alimentar. I gualmente, os artigos categorizados dentro da perspectiva social origi nam-se e/ ou atendem às finalidades da Seção de Pesquisas Econômico-Sociais da referida instituição (Arquivos Brasileiros de Nutrição, 1946:71-73).

Em seguida, na tentativa de garantir inteligibilidade à nossa análise, os artigos da perspectiva biológi ca foram classificados em quatro campos disciplinares ou temáticos da nutrição: nutrição básica e experimental; ciência e tecnologia de al imentos; nutrição clínica (incluindo fisiologia, patologia e dietoterapia) e nutrição e dietética. De forma semelhante, os artigos da perspectiva social foram classificados em outros cinco campos disciplinares ou temáticos da nutrição: avaliação nutricional de populações; política e planejamento em alimentação e nutrição; epidemiologia nutricional; determinantes do estado nutricional de populações e educação nutricional. Tal classificação corresponde a um constructo metodológico que estabelecemos baseados na atual conformação desses campos disciplinares ou temáticos no interior da nutrição.

Os resultados desse estudo são apresentados sob a forma de tabelas de distribuição de freqüência dos atributos investigados. Assim, para cada um destes atributos, foi construído um índice descritivo (número de artigos por volume; número de artigos por natureza da pesquisa e volume; número de páginas por artigo; número de autores por artigo; apresentação de referências bibliográficas por natureza da pesquisa; vínculo institucional do(s) autor(es) por artigo; identidade profissional do autor principal por artigo etc.), que será analisado na seqüência de apresentação dos resultados.

A trajetória dos Arquivos Brasileiros de Nutrição: volumes, número de artigos, periodicidade e direção

A Tabela 1 apresenta a distribuição dos 209 artigos originais analisados conforme o volume, bem como a indicação do ano e o critério de periodicidade de circulação da revista. Mediante a análise do índice número de artigos por volume, observa-se que, ao longo do eixo longitudinal de edição da revista (1944 a 1968), foram publicados, em média, 8,7 artigos por 
Tabela 1

Distribuição dos artigos originais publicados nos Arquivos Brasileiros de N utrição segundo o volume (ano/periodicidade) do periódico, 1944-1968.

\begin{tabular}{|c|c|c|c|}
\hline Volume (ano/periodicidade) & No de artigos & $\%$ & $\%$ acumulado \\
\hline 1 (1944/mensal) & 8 & 3,8 & 3,8 \\
\hline 2 (1946/mensal) & 3 & 1,4 & 5,2 \\
\hline 3 (1947/mensal) & 6 & 2,9 & 8,1 \\
\hline 4 (1947/mensal) & 10 & 4,8 & 12,9 \\
\hline 5 (1948/bimestral) & 6 & 2,9 & 15,8 \\
\hline $6(1949 /$ bimestral $)$ & 14 & 6,7 & 22,5 \\
\hline 7 (1950/bimestral) & 11 & 5,3 & 27,8 \\
\hline 8 (1951/bimestral) & 10 & 4,8 & 32,6 \\
\hline 9 (1953/trimestral) & 5 & 2,4 & 35,0 \\
\hline 10 (1954/trimestral) & 4 & 1,9 & 36,9 \\
\hline 11 (1955/semestral) & 2 & 1,0 & 37,9 \\
\hline 12 (1956/semestral) & 2 & 1,0 & 38,9 \\
\hline 13 (1957/semestral) & 3 & 1,4 & 40,3 \\
\hline $14(1958 /$ semestral) & 6 & 2,9 & 43,2 \\
\hline 15 (1959/semestral) & 9 & 4,3 & 47,5 \\
\hline 16 (1960/semestral) & 12 & 5,7 & 53,2 \\
\hline 17 (1961/semestral) & 9 & 4,3 & 57,5 \\
\hline 18 (1962/anual) & 11 & 5,3 & 62,8 \\
\hline 19 (1963/semestral) & 18 & 8,6 & 71,4 \\
\hline 20 (1964/semestral) & 10 & 4,8 & 76,2 \\
\hline 21 (1965/semestral) & 15 & 7,1 & 83,3 \\
\hline 22 (1966/semestral) & 12 & 5,7 & 89,0 \\
\hline 23 (1967/semestral) & 13 & 6,2 & 95,2 \\
\hline 24 (1968/anual) & 10 & 4,8 & 100,0 \\
\hline Total & 209 & 100,0 & - \\
\hline
\end{tabular}

volume, entretanto podem ser observados cortes transversais que denotam marcantes oscilações desse índice.

Assim, por exemplo, no período de 1944 a 1954, época em que os Arqui vos eram dirigidos por Josué de Castro, tendo como secretários/ redatores os médicos nutrólogos Pedro Borges (1946 a 1947) e Rubens de Siqueira (1947 a 1953), foram publicados $36,9 \%$ do total de artigos, com uma média de 7,7 artigos por volume. Nesse intervalo, a revista, inicialmente com periodicidade mensal, passou a ter uma circulação bimestral (1948 a 1951) e depois trimestral (1953 a 1954), enquanto registrou-se a interrupção de sua circulação nos anos de 1944 (associada à extinção do STAN) e em 1952 (justificada por motivossuperiores não explicitados).

Entre 1955 a 1967, sob a direção de Pedro Borges, que desde 1954 ocupava o cargo de redator, passou a ter circulação semestral e, após superar a crise dos anos 1952-1958 (quando apresentou os menores índices de publicação de artigos por volume), entrou na fase de auge de publicações, com uma média de 12 artigos por volume, tendo sido publicados $58,3 \%$ do total de artigos neste período.

Em seu último volume, relativo ao ano de 1968, a revista teve como diretor-responsável o médico-nutrólogo Hélio Vecchio Alves Maurício, que, desde 1958, ocupava o cargo de redator-secretário. Neste seu último ano de circulação, assim como em 1962, a sua periodicidade foi anual, com dez artigos publicados. Aliás, é preciso destacar que, em seus últimos volumes, conforme atestam algumas notas explicativas contidas em artigos dos volumes 21 , 23 e 24, o periódico circulou com bastante atraso.

Os artigos originais: natureza da pesquisa, tamanho do artigo e normas bibliográficas

A Tabela 2 reúne a apresentação de dois dos índices construídos para a análise dos artigos dos Arquivos: o número de artigos por natureza da pesquisa e volume e número de páginas por natureza da pesquisa e volume. Em relação ao número de artigos por natureza da pesquisa e volume, observa-se que 134 (64,0\%) dos 209 artigos foram incluídos na perspectiva biológica, contra 75 (36,0\%) associados à perspectiva social. Quanto ao número de páginas por natureza da pesquisa e volume, os resultados são semelhantes, apontando um total de $61,0 \%$ de páginas para a perspectiva biológica, contra $39,0 \%$ para a perspectiva social.

No corte inicial (1944 a 1954), correspondente à direção Josué de Castro, a perspectiva social detém apenas 30,0\% dos artigos e 27,5\% do número de páginas publicadas. Já no corte final (1955 a 1968), apesar da perspectiva biológica continuar com sua hegemonia quantitativa, observa-se um aumento relativo das publicações da perspectiva social, a qual passou a deter $39,4 \%$ dos artigos e $48,3 \%$ do número de páginas publicadas.

A análise desses dois índices demonstra que, no decorrer de toda a série histórica de circulação do periódico, a perspectiva biológica esteve à frente da produção e difusão do saber em nutrição. A perspectiva social, al ém de vir a reboque ao longo da série, não aparece no decorrer de pelo menos cinco volumes da revista $(3,5,12,13$ e 14), com exceção do período 1959-1964, momento em que, quantitativamente, supera a perspectiva biológica. Coincidentemente, este período de auge da perspectiva social, conforme discutiremos adiante, corresponde, em termos histórico-conjuntu- 
Distribuição dos artigos originais publicados nos Arquivos Brasileiros de Nutrição segundo o número de artigos e o número de páginas por natureza da pesquisa (biológica ou social) e volume (ano) do periódico, 1944-1968.

\begin{tabular}{|c|c|c|c|c|c|c|}
\hline \multirow[t]{2}{*}{ Volume (ano) } & \multicolumn{3}{|c|}{$\mathrm{N}$ o de artigos } & \multicolumn{3}{|c|}{$\mathrm{N}$ o de páginas } \\
\hline & biológica & social & total & biológica & social & total \\
\hline 1 (1944) & 5 & 3 & 8 & 49 & 28 & 77 \\
\hline $2(1946)$ & 1 & 2 & 3 & 16 & 55 & 71 \\
\hline 3 (1947) & 6 & - & 6 & 149 & - & 149 \\
\hline $4(1947)$ & 9 & 1 & 10 & 149 & 7 & 156 \\
\hline $5(1948)$ & 6 & - & 6 & 216 & - & 216 \\
\hline $6(1949)$ & 9 & 5 & 14 & 152 & 60 & 212 \\
\hline $7(1950)$ & 6 & 5 & 11 & 122 & 96 & 218 \\
\hline $8(1951)$ & 5 & 5 & 10 & 178 & 146 & 324 \\
\hline 9 (1953) & 4 & 1 & 5 & 78 & 22 & 100 \\
\hline $10(1954)$ & 3 & 1 & 4 & 74 & 35 & 109 \\
\hline 11 (1955) & 1 & 1 & 2 & 8 & 36 & 44 \\
\hline 12 (1956) & 2 & - & 2 & 43 & - & 43 \\
\hline 13 (1957) & 3 & - & 3 & 34 & - & 34 \\
\hline 14 (1958) & 6 & - & 6 & 94 & - & 94 \\
\hline 15 (1959) & 4 & 5 & 9 & 36 & 99 & 135 \\
\hline $16(1960)$ & 5 & 7 & 12 & 68 & 118 & 186 \\
\hline 17 (1961) & 5 & 4 & 9 & 63 & 85 & 148 \\
\hline $18(1962)$ & 5 & 6 & 11 & 60 & 121 & 181 \\
\hline 19 (1963) & 12 & 6 & 18 & 150 & 122 & 272 \\
\hline $20(1964)$ & 5 & 5 & 10 & 56 & 111 & 167 \\
\hline 21 (1965) & 10 & 5 & 15 & 117 & 65 & 182 \\
\hline 22 (1966) & 6 & 6 & 12 & 75 & 93 & 168 \\
\hline 23 (1967) & 9 & 4 & 13 & 140 & 42 & 182 \\
\hline 24 (1968) & 7 & 3 & 10 & 72 & 57 & 129 \\
\hline Total & 134 & 75 & 209 & 2.199 & 1.398 & 3.597 \\
\hline$\%$ & $(64,0)$ & $(36,0)$ & $(100,0)$ & $(61,0)$ & $(39,0)$ & $(100,0)$ \\
\hline
\end{tabular}

rais, a um momento de grande mobilização social no País (movimento das Ligas Camponesas; movimentos urbanos pelas reformas de base, incluindo a reforma agrária etc.) e que culmina no golpe militar de março de 1964 (Alencar et al., 1979; Azevêdo, 1982; Vieira, 1983).

Na Tabela 3, encontra-se a distribuição do índice número de páginas por artigo, utilizado aqui como um dos demonstrativos da inexistên cia de rigi dez nos critérios ou condições de publicação do periódico. Na distribuição desse índice, observou-se uma média de cerca de 17, um mínimo de quatro e um máximo de 64 páginas por artigo. Apesar de a grande maioria dos artigos estar abaixo de 25 páginas, observamos que o não-estabel ecimento de um limite máximo de páginas por artigo fez com que os Arqui vos publicassem verdadeiros tratados de fisiopatologia nutricional ou de ciência e tecnologia de alimentos, a exemplo de alguns artigos, como os de Siqueira \& Vogel (1948a, b, c), que perfazem um total de 150 páginas; os de Moura-Campos et al. (1951a e b), que perfazem um total de 104 páginas; e os de Orsini (1947a, b), com 92 páginas.

Em relação à distribuição do índice apresentação de referências bibliográficas por natureza da pesquisa, observamos que 68 (32,5\%) dos 209 artigos publicados não apresentam referências bibliográficas; destes, 40 (58,8\%) são da perspectiva social e $28(41,2 \%)$, da perspectiva biológica. Além dessas evidências, observamos que muitos dos artigos com referências bibliográficas não as apresentam dentro das normas indicadas pelos Arquivos (Index Medicus). Na seqüência de análise dos aspectos das condições redatoriais que contribuem para a demonstração do rigor metodológico da revista, observamos que os artigos da perspectiva biológica, em sua ampla maioria, encontramse dentro da estrutura básica tradicional de publicações periódicas (introdução, metodologia, 
resultados, conclusões, referências, resumos), enquanto os artigos da perspectiva social apenas em sua minoria seguem essa estrutura.

Os autores dos Arquivos: identidade profissional, vínculo institucional e outras características

Na Tabela 4, apresentamos os dados da distribuição do índice número de autores por artigo, onde se observa que 123 (58,9\%) dos artigos são de autoria individual; 50 (23,9\%) são de autoria dupla; $33(15,8 \%)$ são assinados por três ou mais autores e $3(1,4 \%)$, por autores institucionais.

Os 209 artigos originais publicados nos Arquivos são assinados por um total de 134 diferentes autores, 91 dos quais foram considerados autores principais (primeiro nome, no caso de mais de um autor) e 43, autores secundários ou colaboradores. Para a construção do índice identidade profissional do(s) autor(es) por

Tabela 3

Distribuição dos artigos originais publicados nos Arquivos Brasileiros de N utrição segundo o número de páginas por artigo, 1944-1968.

\begin{tabular}{lrrr}
\hline N o de páginas & $\mathrm{n}$ & $\%$ & $\%$ acumulado \\
\hline 4 a 10 & 77 & 36,8 & 36,8 \\
11 a 20 & 79 & 37,8 & 74,6 \\
21 a 30 & 23 & 11,0 & 85,6 \\
31 a 40 & 20 & 9,6 & 95,2 \\
41 a 50 & 5 & 2,4 & 97,6 \\
51 a 64 & 5 & 2,4 & 100,0 \\
Total & 209 & 100,0 & -
\end{tabular}

Tabela 4

Distribuição dos artigos originais publicados nos Arquivos Brasileiros de Nutrição segundo o número de autores por artigo, 1944-1968.

\begin{tabular}{lrr}
\hline No de autores & $\mathrm{n}$ & $\%$ \\
\hline 1 & 123 & 58,9 \\
2 & 50 & 23,9 \\
3 & 24 & 11,5 \\
4 & 5 & 2,4 \\
5 & 3 & 1,4 \\
10 & 1 & 0,5 \\
Institucional & 3 & 1,4 \\
Total & 209 & 100,0 \\
\hline
\end{tabular}

artigo (considerada aqui formação básica), tomamos como referência apenas os 88 autores principais (excluídos os três autores institucionais). Apesar da falta de informações acerca da formação básica de boa parcela destes autores, o que limita bastante a análise desse índice, a distribuição dos dados obtidos é a seguinte: 39 $(44,3 \%)$ dos autores são médicos; oito $(9,1 \%)$ são químicos ou farmacêutico-bioquímicos; cinco $(5,7 \%)$ são nutricionistas; três $(3,4 \%)$ são engenheiros agrônomos; três (3,4\%) são médicos veterinários; um é assistente social; um é economista; seis são autores estrangeiros (convidados especiais dos diretores da revista) e de 22 (25,0\%) não obtivemos informações. Podese afirmar, portanto, que o perfil do autor dos Arquivos apresenta as seguintes características: homem, médico-nutrólogo, inserido na perspectiva biológica da nutrição. Características que, apesar da incorporação de novos elementos, permanecem hegemônicas ao longo da trajetória da revista.

De acordo com o índice vínculo institucional do(s) autor (es) por artigo, os resultados observados para os 209 artigos originais foram os seguintes: 95 (45,5\%) são assinados por autores vinculados ao Inub; 38 (18,2\%), por autores vinculados a outras instituições local izadas no Rio de Janeiro, quase sempre com forte articulação com o grupo de pesquisadores dos Arquivos (a exemplo do Serviço de Alimentação da Previdência Social - Saps - e da Comissão Nacional de Alimentação - CNA - , institui ções também ideal izadas por Josué de Castro) (Coimbra et al., 1982; L'Abbate, 1988); 45 (21,5\%), por autores vinculados a órgãos da Universidade de São Paulo (USP) e de outras instituições deste Estado; e 31 (14,8\%), por autores vinculados a instituições de outros estados brasileiros (Bahia, Minas Gerais, Alagoas e Pernambuco), a instituições internacionais (Organização para a Alimentação e Agricultura - FAO - e Organização Mundial da Saúde - OMS) e alguns não identificados. Dessa forma, às características do perfil do autor dos Arqui vos apontadas acima, acrescentamos: estar vinculado ao I nub e residir no Rio de Janeiro. Características estas que se justificam tanto pelo papel desempenhado pela cidade do Rio de Janeiro (Distrito Federal na época; centro político-intelectual; centro concentrador de três das seis escolas de nutrição existentes até então no País e sede das principais agências estatais da política de alimentação e nutrição), quanto pelo papel desempenhado pelo grupo de médicos nutrólogos do Inub naquele período da trajetória de consolidação do campo da nutrição no Brasil (Coimbra et al., 1982; L'Abbate, 1982; Bosi, 1988). 
Relação dos vinte principais autores dos artigos originais publicados nos Arquivos Brasileiros de Nutrição segundo formação básica, vínculo institucional, campo disciplinar e número absoluto e percentual de artigos publicados em relação ao total, 1944-1968.

\begin{tabular}{|c|c|c|c|c|c|}
\hline $\mathrm{N}$ o de ordem/nome & Formação & Instituição* & Campo & No de artigos & $\%$ do total \\
\hline 1 Pechnik, E. & Q uímica & Inub & Biológico & 42 & 20,2 \\
\hline 2 G uimarães, L. R. & Químico & Inub & Biológico & 21 & 10,1 \\
\hline 3 Borges, P. & Médico & Inub & Social & 17 & 8,2 \\
\hline 4 Siqueira, $\mathrm{R}$. & Médico & Inub & Biológico & 15 & 7,2 \\
\hline 5 Maurício, H. V. A. & Médico & Inub & Social & 10 & 4,8 \\
\hline 6 Silva, W. & Médico & Inub/CNA & Social & 9 & 4,3 \\
\hline 7 Moura-Campos, F. A. & Médico & USP & Biológico & 9 & 4,3 \\
\hline 8 Mucciolo, P. & Med. Veterinária & USP & Biológico & 7 & 3,4 \\
\hline 9 Fioravanti, I. & Médica & Inub & Social & 7 & 3,4 \\
\hline 10 Chaves, J. M. & Q uímico & Inub & Biológico & 7 & 3,4 \\
\hline 11 Schneider, I. S. & Med. Veterinária & USP & Biológico & 6 & 2,9 \\
\hline 12 C astro, J. & Médico & Inub & Social & 5 & 2,4 \\
\hline 13 Dutra-de-O liveira, J. E. & Médico & USP & Biológico & 5 & 2,4 \\
\hline 14 Maldonado, G. F. & Médico & Inub/Saps & Biológico & 5 & 2,4 \\
\hline 15 Ferreira Lima, J. & Médico & Ascofam/PE & Social & 4 & 1,9 \\
\hline 16 Luz, H. S. & Médico & Inub & Biológico & 4 & 1,9 \\
\hline 17 Azevedo, T. & Médico & DS/Bahia & Social & 3 & 1,4 \\
\hline 18 Parahim, 0. & Médico & $\mathrm{DSP} / \mathrm{PE}$ & Social & 3 & 1,4 \\
\hline 19 Lopes, N. & Médico & Ufal/AL & Social & 3 & 1,4 \\
\hline 20 Santos, W. & Médico & Inub/CNA & Social & 3 & 1,4 \\
\hline
\end{tabular}

* Inub: Instituto de N utrição da Universidade de Brasília; CNA: Comissão N acional de Alimentação; USP: Universidade de São Paulo; Saps: Serviço de Alimentação da Previdência Social; Ascofam: Associação Mundial de Luta contra a Fome; DS: Departamento de Saúde; DSP: Departamento de Saúde Pública; Ufal: Universidade Federal de Alagoas.

A Tabela 5 apresenta a relação dos vinte principais autores de acordo com o número de artigos publicados, constituindo-se em uma síntese do perfil de autoria discutido nesta seção. Observa-se que os químicos Emília Pechnik e Luiz Ribeiro Guimarães, técnicos da Seção de Pesquisas Biológicas do Inub, lideram em primeiro e segundo lugares, respectivamente, a lista de publicações. Os médicos nutrólogos do Inub Pedro Borges, Rubens de Siqueira e Hélio Vecchio Alves Maurício, que no decorrer do corte longitudinal 1944-1968 revezaram-se nos cargos de secretário, redator e diretor do periódico, ocupam os postos de terceiro, quarto e quinto lugares, respectivamente. Os cientistas da USP Franklin A. de Moura Campos, Paschoal Mucciolo, I. S. Schneider e José Eduardo Dutra de Oliveira, todos vinculados à perspectiva biológica, assumem os postos de sétimo, oitavo, 11o e 13o lugares, respectivamente. Josué de Castro, em razão de seu papel na criação e direção da revista e na trajetória de constituição do campo da nutrição no Brasil, neste caso, ostenta apenas a 12a posição. Destacamos, ainda, a presença dos médi- cos nutról ogos nordestinos Jamesson Ferreira Lima (Associação Mundial de Luta Contra a Fome - Ascofam/PE), Thales de Azevedo (Departamento de Saúde - DS/ Bahia), Orlando Parahim (Departamento de Saúde Pública - DSP/ PE) e Nabuco Lopes (Universidade Federal de Alagoas - Ufal/ AL), todos vinculados à perspectiva social.

O fato de a cientista Emília Pechnick (na condição de mulher e de química) ocupar, de forma isolada, o primeiro lugar da lista de publicações é tão surpreendente e esclarecedor, quanto contrário a algumas constatações que temos apontado até aqui. Por um lado, indica um certo rompimento com as teses de que a constituição do campo da nutrição no Brasil tenha sido “demarcada por um espaço de luta competitiva pelo monopólio da autoridade científica" (Bourdieu, 1994:131) entre os gêneros masculino e feminino (Santos, 1988; Prado, 1993; Bosi, 1995; Lima, 1997). Sem dúvida, a existência dos 42 artigos assinados pela química Emília Pechnik nos volumes 2 ao 24 da revista indica que, apesar de majoritária, a presença do gênero masculino na trajetória da 
constituição deste campo não é uma condição exclusiva. Por outro lado, reforça as teses da importância da química, considerada como uma das disciplinas-matriz na conformação deste campo, tanto no cenário un iversal, como no Brasil (Chaves, 1978; Lima, 1997; MagaIhães, 1997).

Além dos vinte autores listados, é preciso fazer referência a um grupo que emerge apenas a partir dos anos 60, denotando uma certa abertura dos Arquivos para a publicação da produção científica de outras especialidades que entravam em jogo na contraditória dinâmica de competição e interação que se estabelecia na trajetória de consolidação do campo da nutrição no Brasil. Assim, Enilda Lins da Cruz Gouveia (Saps/RJ) e Maria de Lourdes Mello (Sesi/MG) foram as duas primeiras nutricionistas a publicarem nos Arquivos, cada uma assinando um artigo dentro da perspectiva social (Gouveia, 1960; Mello, 1960). A partir de então, outras nutricionistas passaram a assinar artigos dentro das duas perspectivas que investigamos, a exemplo de Lieselotte H. Ornellas (Escola de Enfermagem da Universidade do Brasil - UB), de Neuza Therezinha Rezende Cavalcante (Inub), de Mirtila C. Araújo (Inub) e de Gilda Linhares Mello (Inub). Referimos ainda a entrada em cena do médico pernambucano Nelson Chaves, fundador do Instituto e do Curso de Nutrição da Universidade Federal de Pernambuco, que, entre 1963 e 1964, assina dois artigos naquele periódico. Fato que, a nosso ver, significa mais um reforço à perspectiva biológica, particularmente às discipl inas fisiologia e endocrinologia, também consideradas disciplinas-matriz na conformação do campo da nutrição no Brasil (Lima, 1997; Magalhães, 1997).

A perspectiva biológica: dos métodos de determinação do valor nutricional aos métodos de industrialização dos alimentos

Nesta seção, passaremos à análise do conteúdo temático dos 134 artigos incluídos na perspectiva biológica da nutrição, dentro dos quatro campos temáticos ou disciplinares em que estes foram categorizados.

A temática nutrição básica e experimental, liderada pelos químicos Emília Pechnik, Luiz Ribeiro Guimarães e José M aria Chaves, da Seção de Pesquisas Biológicas do Inub, e pelo médico Franklin A. de Moura Campos, do Departamento de Fisiologia da Faculdade de Medicina da USP, detém a hegemonia dos trabaIhos desta linha, com $61(45,5 \%)$ artigos publi- cados. Estes artigos, frutos de pesquisas laboratoriais (análises químico-bromatológicas ou ensaios biológico-experimentais), têm como ponto comum a utilização de métodos de determinação do val or nutricional dos alimentos nacionais. Sendo assim, abordam a composição químico-nutricional, o valor calórico, o teor vitamínico e/ ou o teor mineral dos mais diversificados alimentos brasileiros, dos mais tradicionais (feijão preto, farinha de mandioca, trigo, milho, leite, laranjas, manga etc.) aos mais exóticos (buriti, tucumã, pupunha, óleo de patauá, pinhão, mucunã, macambira, sururu etc.). Josué de Castro assina dois artigos do campo temático em questão, um no qual são revelados os resultados químico-analíticos de alimentos bárbaros do sertão nordestino (Castro et al., 1947), e outro que apresenta o valor nutritivo da mistura de milho com leite (Castro \& Pechnik, 1951).

Esse fato representa um dos exemplos que nos ajudam a atestar que não existiam fronteiras rígidas entre as duas perspecti vas investigadas, nem tampouco entre campos temáticos ou disciplinares dentro de uma mesma perspectiva. Vários autores dos Arquivos circulam entre as duas perspectivas e entre os distintos campos temáticos existentes no interior de cada uma delas, ocorrendo, inclusive, tentativas concretas de conexão entre o biológico e o social, a exemplo de artigo incluído nessa temática (Pechnik et al., 1950), no qual os autores apresentam os resultados de uma investigação sobre a castanha-do-pará, investigação esta composta por um detalhado estudo econômico-social da produção e por um ensaio biológico-experimental do valor nutricional deste produto.

A temática tecnologia dos alimentos aparece em segundo lugar dentro da perspectiva biológica, em 35 (26,1\%) dos artigos publicados. Trata-se da única temática alheia aos pesquisadores do Inub, uma vez que quase a totalidade destes artigos são oriundos de pesquisadores do Estado de São Paulo. Paschoal Mucciolo e I. S. Schneider, da Faculdade de Medicina Veterinária da USP, são os dois principais autores dessa linha, cujos artigos abordam os mais diversificados temas ligados à industrialização de alimentos (métodos de processamento, de enriquecimento, de conservação, de embalagem, de controle de qualidade etc.). Embora apareça, de forma bastante tímida, desde 1947, esta temática só teve uma melhor expressão numérica a partir de 1954

A temática nutrição clínica aparece em terceiro lugar, com 25 (18,7\%) artigos publicados. Os médicos Rubens de Siqueira, Hélio de Sou- 
za Luz, Clementino Fraga Filho (da Seção de Patologia da Nutrição do Inub), José Eduardo Dutra de Oliveira e Demosthenes Orsini (da Faculdade de Medicina da USP) são os principais autores dessa linha, cujo auge de produção verificou-se no período de 1947 a 1953, com 16 $(64,0 \%)$ artigos. Os artigos desta área abordam aspectos de fisiologia, patologia e dietoterapia; originaram-se de pesquisas do tipo experimental (ensaios biológicos) e estudos de casos (ensaios clínicos), tratando dos mais diversos temas, tais como semiótica das hipovitaminoses; metabolismo basal; dietoterapia em diabetes, disfunções hepáticas e gastrectomias e padronização de dietas hospitalares.

A temática nutrição e dietética aparece em último lugar, em 13 (9,7\%) dos artigos publicados. Os arti gos desta linha abordam temas relacionados a aspectos do planejamento e administração de serviços de alimentação, da técnica dietética e culinária e da dietética propriamente dita. Quase a totalidade destes artigos $(92,0 \%)$ foram publicados entre 1959 a $1968 \mathrm{e}$ versam, em sua maioria, sobre o planejamento da alimentação de grupos militares (exército, marinha e aeronáutica). Entre os seus autores, destacamos os médicos nutrólogos Pedro Borges, Hélio Vecchio A. Maurício e Irma Fioravanti (Seção de Pesquisas Econômico-Sociais do Inub); Geraldo Francisco Maldonado (Saps) e a nutricionista Liesel otte H. Ornellas (Escola de Enfermagem da UB).

Em síntese, através dos conhecimentos objetivos produzidos por estas pesquisas laboratoriais e ensai os biotecnológicos sobre a composição e o metabolismo dos alimentos; sobre os métodos de industrialização e enriquecimento dos alimentos e sobre os modernos princípios da dietética e da dietoterapia, os médicos nutrólogos vinculados aos Arquivos construíram uma importante base cognitiva para a legitimidade da tese, atribuída a Josué de Castro, de que "a partir deuma al imentação racional, seria possível valorizar a raça,construir o homem brasilei ro e forjar a nação" ( Lima, 1997; Magalhães, 1997:79).

A perspectiva social: da eugenia através da alimentação racional à indústria alimentar no combate à fome

Os 75 artigos incluídos na perspectiva social da nutrição foram categorizados dentro dos cinco campos temáticos ou disciplinares que discutiremos a seguir.

No campo temático Avaliação Nutricional de Populações (que compreendemos como o uso de métodos antropométricos, clínicos, laboratoriais, dietéticos, demográficos e sócioeconômicos para a realização de diagnósticos nutricionais de comunidades), foram incluídos 21 (28,0\%) dos artigos, destacando-se:

- Nove artigos sobre consumo alimentar, utilizando distintas modalidades de inquéritos dietéticos. Tais artigos, publicados do primeiro ao último volume da revista, demonstram que, após o inquérito pioneiro: As condições devida das classes operárias no Recife, realizado por Josué de Castro em 1933 (Castro, 1935), esta modalidade de investigação, além de aprimorarse enquanto instrumental básico de pesquisa dentro da perspectiva social da nutrição, ganhou adeptos em todo o país, a exemplo das pesquisas realizadas pelos médicos nutrólogos Orlando Parahim e Jamesson Ferreira Lima, em Pernambuco; Thales de Azevedo, na Bahia; Oswaldo Costa e Rubem de Siqueira, no Rio de Janeiro; Domingos Lopes e Benedito Philadélpho Siqueira, em Minas Gerais; Nabuco Lopes, em Alagoas, eWalter Silva, no Rio Grande do Norte. - Oito artigos sobre avaliação nutricional baseada em métodos antropométricos, clínicose sócio-econômicos. Ressalte-se que tais avaliações clínico-antropométricas tiveram como diretrizes comuns: a população em idade escolar; algum critério de estratificação sócio-econômica desta população; a utilização de procedimentos estatísticos na amostragem e análise dos dados e resultados apontando melhores perfis antropométrico-nutricionais entre os escolares com maior poder aquisitivo. Estes artigos, publicados entre 1949 a 1968, são assinados pelos médicos nutrólogos Josué de Castro, Walter Silva, Pedro Borges, Thales de Azevedo, Irma Fioravanti, Nabuco Lopes e Geraldo Francisco Maldonado.

No campo temático Política e Planejamento em Ali mentação e Nutrição, foram incluídos 20 (26,7\%) dos artigos. Esta temática emerge em setembro de 1947, a partir de um artigo de Walter Silva que apresenta uma abordagem teórica sobre os três grupos de fatores a serem considerados na formulação de uma política alimentar para o Brasil (disponibilidade de alimentos, poder aquisitivo e capacidade de seleção de alimentos). A articulação destes três grupos de fatores apontados por Silva (1947), no nosso entender, caminhará para a formulação da matriz teórico-conceitual sobre a problemática alimentar e nutricional brasileira, que unificará os intelectuais do campo da nutrição em torno do seu criador, Josué de Castro (Lima, 1997; Magalhães, 1997). Ainda, no nosso entender, implicitamente, através do referido artigo (Silva, 1947), os médicos nutrólogos vin- 
culados aos Arqui vos buscavam gerar consenso e legitimidade para a regulamentação da Comissão Nacional de Alimentação (CNA), agência criada em 1945, mas que só passaria a operar, efetivamente, a partir de 1951, com o retorno de Getúlio Vargas à Presidência da República (L'Abbate, 1982). Aliás, a maioria destes artigos são de natureza teórico-conceitual, apresentando enfoques e propostas políticas sobre temas emergentes em determinadas conjunturas. Como exemplo, podemos apontar o artigo Plano de política alimentar na Amazônia (Castro, 1951), um relatório técnico, no qual Josué de Castro, dentro da abordagem geográfico-ecológica que caracterizará a sua obra (Magalhães, 1997; Lima, 1997), já àquela época, sugeria a implantação de medidas de intervenção alimentar-nutricional visando ao desenvolvimento econômico sustentável para aquela região brasileira, que estava em evidência desde o início da Segunda Guerra Mundial. Destacamos a publicação, no período 1961-1963, de cinco artigos apresentando propostas de produção, abastecimento de alimentos e de reforma agrária (Maurício, 1961; Ferreira-Lima \& Bancovsky, 1962 e 1963; Ferreira Lima et al., 1962; Salomão \& Gomes da Silva, 1963), quando o Brasil inteiro, particularmente a região Nordeste, vivenciava uma intensa mobilização dos trabalhadores rurais com a criação das Ligas Camponesas e dos Sindicatos Rurais (Azevêdo, 1982). Destacamos, ainda, no período 1951-1968, a publicação de quatro artigos abordando a questão da merenda escolar; a nosso ver, foram importantes veículos de difusão das idéias em torno da utilidadeda política nacional de alimentação escolar, implantada no Brasil, no decorrer dos anos 50, a partir dos programas internacionais de ajuda alimentar (Coimbra et al., 1982). E, finalmente, os artigos que prenunciavam o futuro envolvimento que teria a indústria de alimentos na formulação e condução da política de alimentação e nutrição do País no pós-1964.

No campo temático Epidemiologia Nutricional (que compreendemos como o estudo das doenças nutricionais em uma determinada população), foram identificados apenas sete (9,3\%) dos artigos, sendo: a) quatro sobre bócio endêmico, publicados entre 1944 a 1959, originados das atividades desenvolvidas pelo então Departamento Nacional de Saúde, que relatam estudos de prevalência entre escolares ou apresentam revisões sobre prevalência, áreas endêmicas, profilaxia e tratamento da doença. Seus autores são os médicos Álvaro Lobo Leite Pereira, Amílcar Barca Pellon, Walter Silva e Pedro Borges; b) dois sobre desnu- trição protéico-calórica (DPC), de autoria dos médicos Irma Fioravanti e Nelson Chaves, que tratam, respectivamente, de um estudo de prevalência sobre DPC hospitalar e de uma preleção conferida pelo professor, abordando aspectos epi demi ológicos desta carência nutricional no Nordeste; c) um artigo sobre hipovitaminose A, de autoria de Thales de Azevedo; trata-se de uma curta revisão bibliográfica, apresentando alguns dados sobre a prevalência desta carência nutricional na Bahia.

No campo temático Educação Nutricional, foram classificados sete (9,3\%) dos artigos, entretanto apenas três são especificamente fiéis a este campo disciplinar: uma extensa revisão bibliográfica sobre a temática educação nutricional no Brasil (à época denominada Educação Alimentar), publicada em 1951 pelo oficial médico do exército e nutrólogo Walter José dos Santos; um relato da experiência da nutricionista Maria de Lourdes Mello (Sesi/MG) em educação alimentar para menores trabalhadores e um esboço de projeto de pesquisa sobre tabus e hábitos alimentares no Brasil, de autoria do economista Souza Barros (Sesi/Ascofam). Os quatro demais estão relacionados à formação de recursos humanos (nutricionistas, médicos, tecnólogos de alimentos e veterinários), sendo incluídos neste grupo por ser esta a temática mais semelhante. Nesse aspecto, é importante registar que esses trabalhos acerca da formação de recursos humanos, publicados a partir de 1963, parecem datar o acirramento da discussão em torno da demarcação dos campos de saber, de competência e de poder entre os profissionais (especialidades) do campo da nutrição. Nesse sentido, em 1963, foi publicado um artigo sobre tecnologia de alimentos na formação do veterinário (Mucciolo, 1963); a revista relativa ao primeiro semestre de 1966 publicou um artigo sobre o ensino de nutrição para estudantes de Medicina (Dutrade-Oliveira, 1966) e, ainda no segundo semestre de 1966 e em 1967, foram publicados dois artigos sobre a formação do profissional nutricionista (Cavalcante, 1966, 1967).

No campo temático Determinantes do Estado Nutricional de Populações, foram incluídos $20(26,7 \%)$ dos artigos. Apesar de implícito nos artigos da perspectiva social desde 1944, este campo temático só ganhou especificidade a partir de 1950 e expressão numérica nos anos 60. Os artigos nele incluídos, de natureza teórico-conceitual, tratam das concepções ou pontos de vista de distintos autores acerca da gênese, reprodução e conseqüências da problemática alimentar e nutricional no Brasil. Esta politização observada nos artigos ao longo da 
série histórica 1944-1968, a nosso ver, pode ser atribuída à articulação de distintos elementos na trajetória dos intelectuais vinculados aos Arquivos, tais como a maior liberdade de expressão que, gradativamente, foi sendo ampliada no País a partir da queda do Estado Novo; a incorporação de novas concepções científicas e filosóficas, a exemplo, entre outras, da teoria do círculo vicioso da pobreza e da doença no início dos anos 50 (Braga \& Paula, 1981); das teorias do subdesenvolvimento-desenvolvimento a partir de meados dos an os 50 (Mantega, 1985) e do discurso preventivista dos anos 60 (Arouca, 1975) e, finalmente, a maior autonomia que o campo da nutrição foi conquistando no interior do espaço acadêmico (nas universidades e em outras instituições de ensino e pesquisa), do espaço político-institucional (nas agências e instituições estatais vinculadas à política social de al imentação e nutrição) e no seio da sociedade civil (Coimbra et al., 1982; L'Abbate, 1982; Natal, 1982). Assim como nos demais, este campo temático também reflete aspectos das conjunturas específicas em que os artigos foram produzidos e difundidos pelos Arquivos.

Nesse sentido, é preciso atentar para a emergência de temas específicos em determinados cortes transversais da trajetória da revista. Entre 1944 a 1955, permanecia em evidência a tese da ignorância alimentar do povo brasileiro, presente em todas as camadas sociais, embora com certas distinções de cl asse social (Parahim, 1944; Siqueira, 1950). Portanto, tornava-se fundamental a sedutora proposta da "alimentação racional, como fundamento da vida e condição precípua de saúde, eugenia e progresso" (Parahim, 1944:23), legitimadora da constituição do campo da nutrição e da educação alimentar como um dos seus instrumentais básicos (Lima, 1997). Observa-se que a tese do mal de fome e não de raça, formulada por Josué de Castro nos anos 30 - sua forma de interlocução com os cientistas de outros campos disciplinares que, à época, procuravam desfocar da questão biológica para a questão sóciocultural o preconceito de clima (meio) e de raça que se tinha sobre o povo brasileiro (Lima, 1997; Magal hães, 1997) -, continuava, nos anos 44-55, ecoando entre os intelectuais do campo da nutrição. Entretanto, nesse mesmo contexto, outras teses foram sobrepondo-se a essa anterior, a exemplo da linha que concluía que a questão alimentar estava "mais na dependência do poder aquisitivo que de outro fator qualquer e, na impossi bilidade de agir sobre o salário, impõe-se que os serviços de saú de pública, ao lado das ati vidades de edu cação al imentar, promovam atividades que visem aumentar as disponibilidades de alimentos de alto val or nutritivo" (Costa \& Silva, 1946:16-17).

Entre 1959 a 1963, a discussão assumiu novos contornos, ampliando-se o debate em torno dos determinantes demográficos, econômico-sociais e estruturais, particularmente sobre reforma agrária, produção e abastecimento de alimentos, conforme apontamos anteriormente. Além disso, é preciso registrar que: a) a partir de 1959, com a adesão de novos médicos militares à nutrol ogia (Jair de Matos Montedônio, José João Barbosa e Geral do Francisco Maldonado), começaram a ser publicados artigos sobre nutrição nas forças armadas e b) em 1961 foi publicado um relatório técnico sobre a atuação, no Brasil, do programa Alimentos para a Paz, desenvol vido pelo governo norte-americano, prenunciando o assistencialismo alimentar que vigoraria no País no pós-64 (Coimbra et al., 1982; L'Abbate, 1982).

Por fim, entre 1964 e 1968, a discussão central girou em torno dos temas desenvolvimento econômico, população, al imentação, tecnologia e, principalmente, sobre o papel da indústria al imentar no combate à fome (Ornellas, 1965).

\section{Considerações finais}

A opção metodológica adotada neste estudo a realização de uma investigação sistematizada baseada em princípios da meta-análise (Greenland, 1987; Jenicek, 1989; Riegelman \& Hirsch, 1992; Jones, 1993; Thacker, 1993; Escobar, 1994), buscando sumariar aspectos quanti-qualitativos dos artigos originais publicados nos Arqui vos Brasi lei ros de Nutrição - , apesar de demonstrar-se eficiente na consecução dos objetivos a que nos propusemos, apresenta também as suas limitações. Em primeiro lugar, é preciso destacar que o nosso olhar debruçouse apenas sobre uma das seções da estrutura interna de organização do periódico (a seção de artigos originais), o que não nos autoriza a tirar conclusões sobre a trajetória desta publicação científica como um todo. Em segundo lugar, é preciso registrar que, embora acreditemos que os artigos publicados nos Arquivos sejam representativos do que foi produzido e difundido no campo da nutrição ao longo da série histórica 1944-1968, a inexistência de estudos acerca da produção difundida em outras publicações científicas contemporâneas ao periódico (os Arquivos Brasileiros de Medicina, a Revista Brasileira de Medicina, os Arquivos Brasileiros de Endocrinologia e Metabologia, os Ar- 
quivos de Gastroenterologia, os Arquivos de Bromatologia etc.) não nos autoriza a generalizar as conclusões deste estudo. Sendo assim, deixamos acesa a chama para o trilhar de novas investigações neste campo.

A análise dos 209 artigos originais publicados pelos Arqui vos nos possibilitou a identificação não apenas do elenco de intelectuais que participaram do processo de constituição do campo da nutrição, mas também de como as temáticas por estes abordadas foram sendo transformadas ao longo dos 24 anos de circulação da revista. Grosso modo, podemos dizer que, no período 1944-1954, correspondente à direção Josué de Castro, os estudos sobre a composição química e o valor nutricional de alimentos nacionais, desenvol vidos pelos intelectuais vinculados à perspectiva biológica, aliados aos estudos sobre consumo alimentar e estado nutricional da população brasileira, desenvolvidos pelos intelectuais vinculados à perspectiva social, constituíram a base cognitiva geradora da especificidade do campo da nutrição no Brasil, considerada como disciplina, política social e/ ou profissão. A matriz teórica que implícita ou explicitamente unificou tais intelectuais, "a tese do valor eugênico da alimentação na construção da nação e do homem brasileiro" (Lima, 1997; Magalhães, 1997), a nos so ver, sintetiza a ressonância das concepções de cunho nacionalista que caracterizaram os anos 30-40, no campo da nutrição, no Brasil (Miceli, 1979; Pecaut, 1990; Gomes, 1996). Por outro lado, no período 1955-1968, correspondente à direção Pedro Borges/Hélio Vecchio Maurício, a maior politização dos intel ectuais vinculados aos Arqui vosem suas concepções acerca da gênese, reprodução e enfrentamento da fome/subnutrição da população brasileira, sinaliza, em última instância, a comunhão destes intelectuais com as teorias do nacional desenvolvimentismo dos anos 50-60 (Mantega, 1985).

Finalizando, diríamos que, para nós, ao longo dos seus 24 anos de circulação, os Arquivos parecem ter cumprido os objetivos explíci- tos idealizados por seu diretor-fundador de contribuir com a difusão dos modernos conhecimentos científicos sobre nutrição para a formação de novas gerações de nutrólogos, nutricionistas e outros especialistas deste campo (Castro, 1944). Além desse mérito técnico-científico, é preciso reconhecer a importância dos Arquivos como instrumento político-ideológico a serviço de interesses específicos dos cientistas que os conduziram. A análise da série histórica 1944-1968 nos faz concluir que, mesmo com o afastamento oficial de Josué de Castro da direção da revista, quando o mesmo passou a exercer sua militância política no período de 1955-1964 (ocupando os cargos de deputado federal pelo estado de Pernambuco e de embaixador brasileiro junto à Organização das Nações Unidas - ONU) (Coimbra et al., 1982; L'Abbate, 1982), sob a direção dos seus discípulos mais diletos (Pedro Borges e Hélio Vecchio A. Maurício), os Arquivos mantiveram a mesma diretriz e até dinamizaram quantitativamente o seu número de publicações. Após o golpe militar de 1964, com o exílio de seu diretor-fundador, os Arquivos conseguiram circular até 1968, encerrando, assim, uma importante fase da nossa história, quando "dentro das paredes fechadas dos laboratórios, no campo aberto da pesqui sa social, na cátedra e nos livros, secriou a escola brasileira de Nutrição" (Castro, 1944:3). Fase esta liderada por um grupo de médicos nutrólogos que, ao mesmo tempo em que procuraram estabelecer as conexões entre o biológico e o social, com base na interdisciplinaridadee na complexidade inerentes ao novo campo do saber que se constituía, contraditoriamente, criaram a demarcação de outras fronteiras, definidoras dos limites de competên cia, de autonomia e de poder - na concepção teórica de Bourdieu (1987 e 1994) -, entre as especialidades do campo da nutrição, particularmente entre médicos nutrólogos e nutricionistas. Assim, em 1968, formou-se um hiato para a (re)construção de uma nova escola brasileira de nutrição. Mas isso é uma outra história.

\section{Agradecimentos}

Aos professores Ricardo Ventura Santos e Carlos E. A. Coimbra Jr. (Escola Nacional de Saúde Pública, Fundação Oswal do Cruz), pela prestimosa orientação e leitura crítica do texto original. 
ALENCAR, F.; CARPI, L. \& RIBEIRO, M. V., 1979. História da Soci edade Brasileira. Rio de Janeiro: Ao Livro Técnico.

AROUCA, A. S. S., 1975. O Dilema Preventivista:Contribui ção para a Compreensão e Crítica da Medicina Preventiva. Tese de Doutorado, Campinas: Faculdade de Ciências M édicas, Universidade Estadual de Campinas.

ARQUIVOS BRASILEI ROS DE NUTRIÇÃO, 1946. Instituto de Nutrição (Notase Informações), 2:71-73.

AZEVÊDO, F. A., 1982. As Ligas Camponesas. Rio de Janeiro: Paz eTerra.

BOSI, M. L. M., 1988. A Face Oculta da Nutrição: Ciência el deologia. Rio de Janeiro: Espaço e Tempo/ Editora da Universidade Federal do Rio de Janeiro.

BOSI, M. L. M., 1995. Definindo Fronteiras: O Nutricionista esua Profissionalização. Tese de Doutorado, Rio de Janeiro: Escola Nacional de Saúde Pública, Fundação Oswaldo Cruz.

BOURDIEU, P., 1987. A Economia das Trocas Simbólicas. São Paulo: Perspectiva.

BOURDIEU, P., 1994. El campo científico. Revista de Estudios Sociales dela Ciencia (Redes), 1:131-160.

BRAGA, J. C. S. \& PAULA, S. G., 1981. Saúde e Previdência: Estudos de Política Social. São Paulo: Cebes/Hucitec.

CASTRO, A. M., 1977. Nutrição e Desenvolvimento: Análise de uma Política. Tese de Livre-Docência em Sociologia, Rio de Janeiro: Instituto de Nutrição, Centro de Ciências da Saúde, Universidade Federal do Rio de Janeiro.

CASTRO, J., 1935. As Condições de Vida das Classes Operárias no Recife. Rio de Janeiro: Ministério do Trabalho.

CASTRO, J., 1944. O "Serviço Técnico da Alimentação Nacional" e os "Arquivos Brasileiros de Nutrição". Arquivos Brasileiros de Nutrição, 1:3-6.

CASTRO, J., 1946. Editorial. Arquivos Brasileiros de Nutrição, 2:3-5.

CASTRO, J., 1951. Plano de política alimentar na Amazônia. Arquivos Brasileiros de Nutrição, 8: 195-204.

CASTRO, J.; PECHNIK, E.; PARAHIM, O.; MATTOSO, I. V. \& CHAVES, J. M., 1947. Os "alimentos bárbaros" dos Sertões do Nordeste. Arquivos Brasileiros de Nutrição, 3:5-29.

CASTRO, J. \& PECHNIK, E., 1951. Valor nutritivo da mistura de milho com leite. Arquivos Brasilei ros de Nutrição, 8:13-30.

CAVALCANTE, N.T. R., 1966. O profissional em nutrição: formação, atividades e perspectivas futuras. Arquivos Brasileiros de Nutrição, 22:51-64.

CAVALCANTE, N. T. R., 1967. Preparação do nutricionista face o desenvolvimento. Arqui vos Brasileiros de Nutrição, 23:77-88.

COSTA, O. \& SILVA, W., 1946. Quatro inquéritos sobre hábitos alimentares. Arquivos Brasileiros de Nutrição, 2:6-55.

COIM BRA, M.; MEIRA, J. F. P. \& STARLING, M. B. L., 1982. Comer eAprender: Uma História da Alimentação Escolar no Brasil. Belo Horizonte: Instituto Nacional de Alimentação Escolar, Ministério da Educação e Cultura.
CHAVES, N., 1978. Nutrição Básica e Aplicada. Rio de Janeiro: Guanabara-Koogan.

DUTRA-DE-OLIVEIRA, J. E., 1966. Ensino de nutrição para estudantes de Medicina. Arquivos Brasileiros deNutrição, 22:21-36.

ESCOBAR, A. L., 1994. Malária no Sudeste da Amazônia: Uma Meta-Análise. Dissertação de Mestrado, Rio de Janeiro: Escola Nacional de Saúde Pública, Fundação Oswal do Cruz.

FERREIRA-LIM A, J.; COSTA, F. \& MELLO, F. B., 1962. Algumas medidas para a melhoria do abastecimento regi onal. Arquivos Brasileiros de Nutrição, 18:95-120.

FERREIRA-LIMA, J . \& BANCOVSKY, J ., 1962. O drama da fome no Nordeste. Arquivos Brasilei ros de Nutrição, 18:155-192.

FERREIRA-LIMA, J. \& BANCOVSKY, J., 1963. Plano de alimentação racional. Arquivos Brasilei ros de Nutrição, 19:85-115.

GOMES, A. C., 1996. História eHistoriadores: A Política Cultural do Estado Novo. Rio de Janeiro: Editora da Fundação Getúlio Vargas.

GOUVEIA, E. L. C., 1960. O papel dos inquéritos alimentares nos programas educacionais e assistenciais de coletividades. Arquivos Brasileiros de Nutrição, 16:69-78.

GREENLAND, S., 1987. Quantitative methods in the review of epidemiological literature. Epidemiologic Reviews, 9:1-30.

JENICEK, M., 1989. Meta-analysis in Medicine where we are and where we want to go? Journal of Clinical Epidemiology, 42:35-44.

JONES, D. R., 1993. El metanálisis en los estudios epidemiológicos observacionales. Bol etín dela Oficina Sanitaria Panamericana, 115:438-445.

L'ABBATE, S., 1982. Fome e Desnutrição: Os Descaminhos da Política Social. Dissertação de Mestrado, São Paulo: Departamento de Ciências Sociais da Faculdade de Filosofia, Letras e Ciências Humanas, Universidade de São Paulo.

L'ABBATE, S., 1988. As políticas de alimentação e nutrição no Brasil. I. Período de 1940 a 1964. Revista de Nutrição da PUCCAMP, 1:87-138.

LIM A, E. S., 1997. Gênese eConstituição da Educação Alimentar: A Instauração da Norma. Tese de Doutorado, São Paulo: Pontifícia Universidade Católica de São Paulo.

MAGALHÃES, R., 1997. Fome: Uma (Re)leitura deJosuédeCastro. Rio de Janeiro: Editora Fiocruz.

MANTEGA, G., 1985. A Economia Política Brasileira. Petrópolis: Editora Vozes/ Polis.

MAURíCIO, H. V. A., 1961. Alimentação e reforma agrária, problemas sociais. Arquivos Brasilei ros de Nutrição, 17:17-27.

MELLO, M. L., 1960. A educação alimentar para o menor trabalhador. Arquivos Brasileiros de Nutrição, 16:79-90.

MICELI, S., 1979. Intel ectuais e Classe Dirigente no Brasil (1920-1945). São Paulo: Difel.

MOURA-CAMPOS, F. A.; PECHNIK, E. \& SIQUEIRA, R., 1951a. Valor nutritivo de frutos brasileiros. Arquivos Brasilei ros de Nutrição, 8:99-162.

MOURA-CAMPOS, F. A.; PECHNIK, E. \& SIQUEIRA, R., 1951b. Valor nutritivo de frutos brasileiros (con- 
clusão). Arquivos Brasilei ros de Nutrição, 8:205244.

MUCCIOLO, P., 1963. Tecnologia dos alimentos no curriculum veterinário. Arquivos Brasileiros de Nutrição, 19:29-35.

NATAL, J. L. A., 1982. A Questão Alimentar-nutricional na Política Econômica (1930-1976): Um Vai-vem na Periferia da Agenda Pública. Dissertação de Mestrado, Campinas: Departamento de Economia e Planejamento, Instituto de Filosofia e Ciências Humanas, Universi dade Estadual de Campinas.

ORNELLAS, L. H., 1965. A indústria alimentar no combate à fome. Arquivos Brasileiros de Nutrição, 21:51-64.

ORSI NI, D., 1947a. O metabolismo de base de jovens brasileiras. Arquivos Brasilei ros de Nutrição, 3:636.

ORSINI, D., 1947b. O metabolismo de base de jovens brasileiras (continuação). Arquivos Brasileiros de Nutrição, 3:6-66.

PARAHIM , O., 1944. O problema alimentar no Sertão Nordestino: a eugenia através da alimentação racional. Arquivos Brasilei ros de Nutrição, 1:23-36.

PECAUT, D., 1990. Os Intel ectuais e a Política no Brasil: Entreo Povo ea Nação. São Paulo: Editora Ática.

PECHNIK, E.; BORGES, P. \& SIQUEIRA, R., 1950. Estudo sobre a Castanha do Pará. Arquivos Brasileiros de Nutrição, 7:7-42.

PRADO, S. D., 1993. Autonomia: Tentadora e Reificada. Estudos sobre Nutricioni stas dos Centros Muni cipais de Saúde do Rio de Janeiro. Dissertação de Mestrado, Rio de Janeiro: Escola Nacional de Saúde Pública, Fundação Oswaldo Cruz.

RIEGELMAN, R. K. \& HIRSCH, R. P., 1992. Cómo Estudiar un Estudio y Probar una Prueba: Lectura Crítica de la Literatura Médica. Organización Panamericana de la Salud, Publicación Científica 531. Washington: Organización Panamericana de la Salud.
SALOMÃO, J. P. \& GOMES DA SILVA, J., 1963. Reforma agrária e produção de al imentos. Arquivos Brasi leiros de Nutrição, 19:81-118.

SANTOS, S. M. C., 1988. Nutricionista \& Sociedade Brasileira: Elementos para Abordagem HistóricoSocial da Profissão. Dissertação de Mestrado, Salvador: Universidade Federal da Bahia.

SILVA, W., 1947. Política de alimentação. Arquivos Brasileiros deNutrição, 4:6-12.

SIQUEIRA, R., 1950. Desorganização: problema grave e causa importante de má al imentação de coletividades brasileiras. Arquivos Brasilei ros de Nutrição, 7:379-396.

SIQUEIRA, R. \& VOGEL, J., 1948a. Aspectos semióticos das carências vitamínicas no homem e nos animais domésticos. Arquivos Brasilei ros de Nutrição, 5:5-46.

SIQUEIRA, R. \& VOGEL, J., 1948b. Aspectos semióticos das carências vitamínicas no homem e nos animais domésticos (continuação). Arquivos Brasileiros de Nutrição, 5:5-58.

SIQUEIRA, R. \& VOGEL, J., 1948c. Aspectos semióticos das carências vitamínicas no homem e nos animais domésticos (conclusão). Arquivos Brasileiros de Nutrição, 5:5-58.

THACKER, S. B., 1993. Metanálisis: un enfoque cuantitativo para la integración de investigaciones. Boletín de la Oficina Sanitaria Panamericana, 115:328-339.

VASCONCELOS, F. A. G., 1988. A Política Social deAlimentação e Nutrição no Brasil: Acumulação de Capital e Reprodução da Fome/Desnutrição (do Estado Novo à Nova República). Dissertação de Mestrado, João Pessoa: Departamento de Serviço Social, Centro de Ciências Humanas, Letras e Artes, Universidade Federal da Paraíba.

VIEIRA, E., 1983. Estado e Mi séria Social no Brasil - de Getúlio a Geisel. São Paulo: Cortez. 